\title{
Prevalence and Clinical Features of Human Bocavirus Infection in Hospitalized Pediatric Patients in a Tertiary Hospital in Oman: Retrospective Study
}

\section{Nawal AL Maskari*, Omaira AL Omairi and Hanan AL Kindi \\ Royal hospital, The university of Oman, Oman}

*Corresponding author: Nawal A L Maskari, Royal hospital, The university of Oman, Muscat- Airport, Oman, Tel: 0096899464076; Email: nawal.almaskari@gmail.com

\section{Research Article}

Volume 2 Issue 2

Received Date: May 03, 2018

Published Date: May 31, 2018

\section{Abstract}

Background: "Human bocavirus (HBoV) is a new member of the Parvoviridae family". It has been discovered on 2005 from pediatric patients with acute respiratory tract infections (ARTI). The present study is the first study provides prevalence and clinical presentations of HBoV infection among all Royal Hospital (RH) pediatric patients with ARTI and positive $\mathrm{HBoV}$. This study also investigated $\mathrm{HBoV}$ co-infection and association with the severity of HBoV manifestations.

Methods: This is a retrospective descriptive study reviewed medical records of all pediatric patients hospitalized in RH with $\mathrm{ARI}$ and positive $\mathrm{HBoV}$ respiratory tracts samples (nasopharyngeal aspirates, tracheal secretions and Bronchoalveolar lavages) using polymerase chain reaction assay (PCR) from September 2014 to September 2016. All samples were screened for other viruses by PCR HBoV. Clinical presentations and demographic characteristics were investigated.

Result: HBoV PCR was positive in 111 out of 3309 pediatric patients aged from 0 to 13 years presented with ARTI. HBoV Co-infection rate with other virus has been reported in $58.6 \%$ of children and the most common co-infected virus is respiratory syncytial virus (28\%) followed by rhinovirus (21\%) and adenovirus (17.4\%). 19.8\% of HBoV infected children required pediatric intensive care unit (PICU) admission and 90\% of PICU admission required mechanical ventilation. The median age was 12 month.

Conclusion: $\mathrm{HBoV}$ was detected in $3.35 \%$ of children with ARTI. It mainly affects children less than 2 years of age and mainly presents with ARTI. It has high co-infection rate. There was no association between patient's age, co-infected viruses and severity of clinical presentation, Human, Child, respiratory tract sample, respiratory tract infections, pathogenesis.

Advances in Knowledge: 1- This study evaluates the burden of HBoV infection in pediatric patients.

2- It demonstrates a local epidemiological data, clinical presentations and severity of the infection.

3-It shows the outcome of $\mathrm{HBoV}$ infection as it is a recently discovered member of Parvoviridae family. 


\section{Journal of Infectious Diseases \& Travel Medicine}

Application for the Patient Care: 1-It also reveals the length of hospital stay which has an impact on the social life of the patients and the family.

2-It shows a good outcome of HBoV infection which is important to know when counselling the family.

2-It also emphasizes the need of isolation precaution to prevent the spread of infection to the health care workers and population.

Keywords: Human Bocavirus; Prevalence; Pediatric

Abbreviations: ARTI: Acute Respiratory Tract Infections; PCR: Polymerase Chain Reaction Assay; PICU: Pediatric Intensive Care Unit; HBoV: Human bocavirus; CPHL: Central Public Health Laboratories; HPIV: Human Para Influenza Virus; CXR: Chest X-Ray; HMPV: Human Meta Pneumo Virus.

\section{Introduction}

$\mathrm{HBoV}$ is relatively new virus discovered on 2005 . It is a very small, non-enveloped virus with a single-stranded DNA genome. It has been isolated from the respiratory samples of Swedish pediatric patients with lower respiratory tract infection [1]. It has 4 genotypes HBoV1, 2,3 and 4 [2]. Usually the management is supportive as it is self-limited virus and doesn't require antiviral medication. The prognosis is usually good. It affects mainly children less than 2 years of age, although adult cases have been reported [3]. It mostly manifests as acute respiratory tract infection (ARTI) and may present with extrarpulmonary manifestations like diarrhea [4,5]. It may present with mild or severe respiratory symptoms require intensive care admission [6,7].

The present study is the first study in Oman aimed to investigate the prevalence of Human bocavirus (HBoV) infection in all Royal Hospital hospitalized pediatric patients with positive $\mathrm{HBoV}$. It also analyzes $\mathrm{HBoV}$ clinical presentations and co-infection with other viruses.

\section{Method}

This retrospective descriptive study reviewed the medical records data of all pediatric patients with $\mathrm{HBoV}$ ARTI. The inclusion criteria were all Royal Hospital pediatric patients aged from newborn to 13 years old who were registered in emergency room department (ER), admitted to pediatric wards and pediatric intensive care unit (PICU) diagnosed to have positive $\mathrm{HBoV}$ molecular assay during the period of September 2014 to September 2016. All respiratory tract samples (nasopharyngeal aspirates, tracheal secretions, and bronchoalveolar lavages) were screened for $\mathrm{HBoV}$ and other nine viruses. We excluded other patients with positive RVP for other viruses but negative $\mathrm{HBoV}$.

RVP samples were collected and sent in viral transport media to the virology department at the Central Public Health Laboratories (CPHL). Samples were automatically extracted by Qiagen, QIA symphony machine and HBoV was detected by a multiplex commercial polymerase chain reaction assay (PCR) from Fast Track Diagnostic Respiratory Pathogens 21 Kit (fast-track diagnostics, Junglinster Luxembourg) and the Applied Biosystems ${ }^{\circledR}$ 7500 Real-Time PCR Systems. The RVP kit used to detects the following respiratory pathogens $\mathrm{HBoV}$ (detect 4genotypes in general but cannot differentiate between them), respiratory syncytial virus (RSV); rhinovirus (RV); influenza viruses (A and $B$ ), human para influenza virus (HPIV): 1 to 4, adenovirus; enteroviruses, human metapneumo virus (HMPV), paraecho virus, corona virus. The total screened RVP was 3309 and HBoV PCR was positive in111.

The symptoms and signs of cough, fever, flu-like illness, respiratory distress, with the impression of upper respiratory tract infection, pneumonia, bronchiolitis, and asthma were included. The definition of pneumonia in our study is lung infiltration or patch shown in the chest X-ray (CXR) and fine crackles on auscultation. The severity of illness defined as that patient who required high dependency and intensive care support including invasive and noninvasive ventilation. We excluded other patients with positive RVP for other viruses and negative for $\mathrm{HBoV}$. The severity of symptoms defined as that patient with respiratory distress who required high dependency care and intensive care support including invasive and noninvasive ventilation. 
Then retrospectively we started to collect patients' epidemiological data, clinical presentations, diagnosis, radiological images and condition on discharge through patient medical charts using AL-Shifa computer system from the Royal Portal and the data sheets were filled up. Patient's data were summarized by descriptive statistics. Epi data 3.1 software was used for data entry and Microsoft excel was used in statistical analyses.

Categorical and continuous values were expressed as frequency and percentage.

The medical ethics \& Scientific Research committee approved the study and approval number is MERC\#103/2016.

\section{Results}

HBoV was detected in only 111 pediatric patients out of 3309 with positivity rate of $3.35 \%$ which is the same as other studies reported. Hospitalization was required in (93\%):59.5\% admitted in the ward, $12.6 \%$ in high dependency, $19.8 \%$ required PICU admission and $8.1 \%$ discharged from ER or outpatient clinic. $21.6 \%$ out of 111 required noninvasive respiratory supports and $18.0 \%$ needed mechanical ventilation. The median days of hospital stay was 7 days.80\% of our affected patients were less than 2 years old as shown in Figure 1.

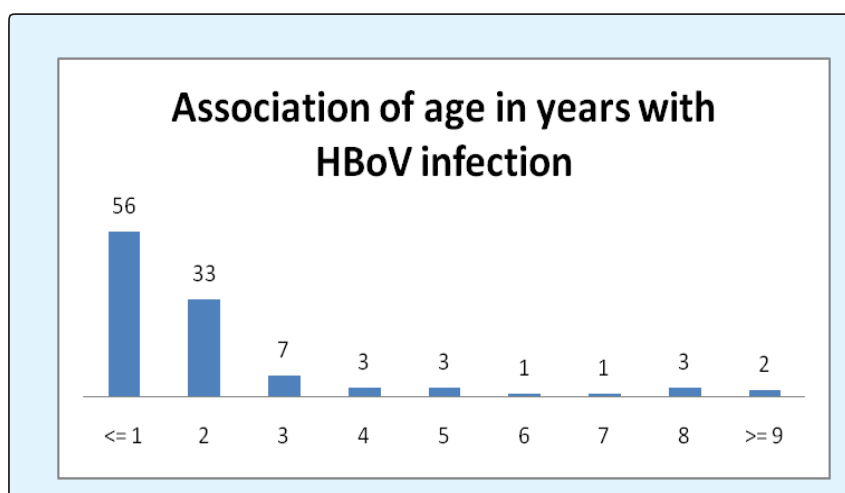

Figure 1: Age distribution of $\mathrm{HBoV}$ infection.

The study showed the median age of affected children is 12 months and no difference between male and female (male: $49 \%$ and female: $51 \%$ ).

The common symptoms were fever (84\%), cough (81\%) followed by flulike illness $(46.8 \%)$. The most common clinical diagnosis was pneumonia (52\%), followed by upper respiratory tract infection $(22.5 \%)$, bronchiolitis (15.3\%) and asthma exacerbation (0.08\%). CXR showed pneumonia in 52\%, normal or not done $41.6 \%$ and peribronchial infiltrates $0.06 \%$.

The rate of co-infections of $\mathrm{HBoV}$ with other respiratory viruses was $58.6 \%$.At least one co-infected virus was detected in $58.6 \%$, double $35.1 \%$ and triple in $0.06 \%$ patients. The most common co-infected viruses are RSV (28\%) followed by rhinovirus (21\%), adenovirus $(17.4 \%)$ enterovirus $(13.0 \%)$, parainfluenza virus $(10.9 \%)$, corona $(10.9 \%)$, influenza $(4.3 \%)$ and HMPV (4.3\%).

\section{Discussion}

The present study is the first study in Oman aimed to investigate the prevalence and the clinical presentations of $\mathrm{HBoV}$ infection among children. The prevalence of $\mathrm{HBoV}$ varies from $1.5 \%$ to $19 \%$ which depend on the age of the patients and seasonal fluctuation. The pathogenesis of $\mathrm{HBoV}$ is still unclear [2]. Some studies reported a strong association between HBoV infection and ARTI and other showed no significant association [8,9]. This study showed $\mathrm{HBoV}$ infection presents mainly with mild to severe ARTI. The current study showed also the majority of $\mathrm{HBoV}$ infected patients were admitted in the ward with ARTI and only $19 \%$ required intensive care admission. It is very difficult to conclude that $\mathrm{HBoV}$ is the primary infection or co-infections as other viruses can present with same findings. Some literatures showed no seasonal variation while other studies showed more of $\mathrm{HBoV}$ infection during winter and spring months [10]. The present study reported that the maximum number of $\mathrm{HBoV}$ infection during spring and autumn season as shown in figure 2 .

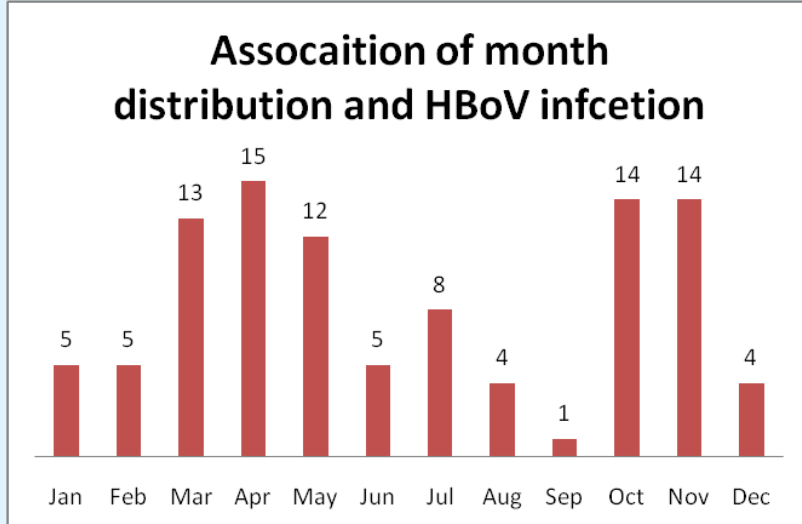

Figure 2: Month distribution of $\mathrm{HBoV}$ infection. 


\section{Journal of Infectious Diseases \& Travel Medicine}

This research showed a co-infection rate was58.6\% and RSV is the most co-infected virus. Other studies reported the same; a co-infection rate was $29 \%$ to $78 \%$ and also RSV is the most co-infected virus. Some studies showed there is no difference in clinical or laboratory results between $\mathrm{HBoV}$ single infection or co-infection with single, double or triple viruses which is similar to the present study $[2,3,8]$.

This study showed the major clinical presentations were fever, cough, flulike illness and wheezing. The most common clinical diagnoses were pneumonia followed by upper respiratory tract infection, bronchiolitis and asthma exacerbation which is similar to other studies [3]. Only one-third of CXR were normal. The high rate of abnormal radiological finding may be explained relatively as most of the patients are young age. This is also supported by other studies.

$50 \%$ of the patients who required PICU admission has single, $36 \%$ double and $4.5 \%$ triple co-infected viruses. This report indicates $\mathrm{HBoV}$ can be associated with severe respiratory infections requiring PICU admission but there is no obvious relationship between a number of coinfected viruses and disease severity. Mortality of 3 children was reported and their death attributed to their primary disease. The age and diagnosis of 3 expired patients was 8 months old infant with biliary atresia, 1 month old with complex heart disease and 12 months old with corrosive ingestion.

\section{Limitation to the Study}

The major limitation to the study is it very difficult to know $\mathrm{HBoV}$ is the primary infection or co-infected with other viruses as other respiratory viruses can present with the same clinical and radiological finding. Other limitation is also detection doesn't mean all of the time infection it can be found in healthy person without host response.

\section{Conclusion}

HBV infection mainly affects children less than 2 years of age and mainly presents with ARTI. It has high coinfection rate. There was no association between patient's age, co-infected viruses and severity of clinical manifestations.

\section{References}

1. Allander T, Tammi MT, Eriksson M, Bjerkner A, Tiveljung-Lindell A, et al. (2005) Cloning of a human parvovirus by molecular screening of respiratory tract samples. Proc Natl AcadSci USA 102(36): 1289112896.

2. Marcello Guido, Maria Rosaria Tumolo, TizianoVerri, Alessandro Romano, Francesca Serio, et al. (2016) Human bocavirus: Current knowledge and future challenges. World J Gastroenterol 22(39): 8684-8697.

3. Chia-Yunn Chuang, Chuan-Liang Kao, Li-Min Huang, Chun-Yi LU, Pei-Lan Shao, et al. (2011) Human bocavirus as an important cause of respiratory tract infection in Taiwanese children. Journal of Microbiology, Immunology and Infection 44(5): 323327.

4. Mei Zeng, Qi-Rong Zhu, Xiao-Hong Wang, Hui Yu, Jun Shen (2010) Humanbocavirus in children with respiratory tract infection in Shanghai: a retrospective study. World J Pediatr 6(1): 65-70.

5. Albuquerque MC, Rocha LN, Benati FJ, Soares CC, Maranhao AG, et al. (2007) Human boccavirus infection in children with gastroenteritis, Brazil. Emerg Infec Dis 13(11): 1756-1758.

6. Lau SK, Yip CC, Que TL, Lee RA, Au-Yeung RK, et al. (2007) Clinical and molecular epidemiology of human bocavirus in respiratory and fecal samples from children in Hong Kong. J Infect Dis 196(7): 986-993.

7. Pozo F, Garcia-Garcia ML, Clavo C, Cuesta I, PerezBrena P, et al. (2007) High incidence of human boccavirus infection in children in Sapin. J Clin Virol 40(3): 224-228.

8. Verena Schildgen, Monika,Malecki, Ramona-Liza Tillmann (2011) Michael Brockman and Oliver Schildgen. The Human Bocavirus Is Associated with Some Lung and Colorectal Cancers and Persists in Solid Tumors. Future Virol 6(9): 1107-1114.

9. Arden KE, McErlean P, Nissen MD, Sloots TP, Mackay IM (2006) Frequent detection of human rhinoviruses, paramyxoviruses, coronaviruses, and bocavirus during acute respiratory tract infections. J Med Virol 78(9): 1232-1240.

10. Chi-Chun Sung, Hsin Chi, Nan -Chang Chiu, Daniel Tsung-Ning Huang, Li-Chuan Weng, et al. (2011) Viral etiology of acute lower respiratory tract infections in hospitalized young children in Northern Taiwan. Journal of microbiology, Immunology and Infection 44(3): 184-190. 